\title{
Respuestas a la sesión de relajación: investigaciones en el deporte
}

\section{Responses to the relaxation session: research in sport}

\author{
* Ana Carolina Lima Costa
}

Lima, A. C. (2021). Respuestas a la sesión de relajación: investigaciones en el deporte. Revista Ciencias de la Actividad Física UCM, 22(2), julio-diciembre, 1-14. http://doi.org/10.29035/rcaf.22.2.9

\section{RESUMEN}

Las técnicas de relajación son recomendadas y utilizadas como estrategia para ayudar al individuo a lidiar con el estrés físico, psicológico y social. Este trabajo procuró conocer el uso de métodos de relajación utilizados en deportistas. Se ha utilizado la revisión bibliográfica basada en la búsqueda, análisis e interpretación de la literatura científica disponible sobre la relación entre las técnicas de relajación y el deporte de competición. El objetivo del estudio ha sido identificar y analizar los posibles efectos de las técnicas de relajación en los deportistas. Para ello, se examinaron los métodos utilizados y las evidencias de eficacia en el proceso de entrenamiento y/o competiciones, y admitieron artículos que utilizaron instrumentos y procedimientos de carácter cuantitativo y cualitativo. Los resultados indican que las técnicas de relajación poseen, en su concepción, beneficios que son útiles no sólo al bienestar del atleta, sino que también contribuyen con su recuperación a nivel físico y psicológico.

Palabras clave: bienestar, rendimiento, competición, deportista.

\section{ABSTRACT}

Relaxation techniques are recommended and used as a strategy to help the individual cope with physical, psychological and social stress. This work sought to know the use of relaxation methods used in athletes. The literature review was used based on the search, analysis and interpretation of the available scientific literature on the relationship between relaxation techniques and competitive sport. The objective of the study has been to identify and analyze the possible effects of relaxation on athletes. To do this, the methods used and the evidence of effectiveness in the training process and / or competitions were examined and admitted articles that used quantitative and qualitative instruments and procedures. The results indicate that relaxation techniques have in their conception benefits that are useful not only to the well-being of the athlete, but also helps in recovery at the physical and psychological level.

Key words: wellness; performance; competition; athlete.

\section{RESUMO}

Técnicas de relaxamento são recomendadas e utilizadas como estratégia para ajudar o indivíduo a lidar com o estrese físico, psicológico e social. Este trabalho buscou conhecer o uso de métodos de relaxamento utilizados em atletas. A revisão da literatura foi utilizada com base na busca, análise e interpretação da literatura científica disponível sobre a relação entre técnicas de relaxamento e esporte competitivo. O objetivo do estudo foi identificar e analisar os possíveis efeitos do relaxamento nos atletas. Para isso, foram examinados os métodos utilizados e as evidências de efetividade no processo de treinamento e / ou competições e admitidos artigos que utilizavam instrumentos e procedimentos quantitativos e qualitativos. Os resultados indicam que as técnicas de relaxamento têm em sua concepção benefícios que são úteis não apenas para o bem-estar do atleta, mas também ajudam na recuperação nos níveis físico e psicológico.

Palavras chave: bem-estar; rendimento; competição; atleta.

* Doctora en Ciencias de la Actividad Física y del Deporte. Secretaria Ejecutiva de Deporte del Estado de Pernambuco. Madrid, España. https://orcid.org/0000-0003-2959-0625 | carolcostabr@gmail.com 


\section{INTRODUCCIÓN}

El nivel de práctica de actividad física de atletas de competición refleja la gran exigencia del entrenamiento. Además, a los atletas súmase los compromisos con la escuela o trabajo, dedicación a la familia y el hogar y amigos. Ante este escenario, las técnicas de relajación constituyen una estrategia que puede ser utilizada para ayudar al individuo a lidiar con el estrés tanto emocional como psicológico. Tradicionalmente, las variadas técnicas tienen como objetivo común producir en el cuerpo una respuesta natural de relajación con una sensación de calma y bienestar, siendo utilizadas para inducir o mejorar la calidad del sueño, reducir el dolor y tranquilizar o controlar las emociones (Calder, 2007, NCCAM, 2011, Swimming/Natation Canada, 2008, Scott et al., 2016, Walsh, 2011).

En el ámbito deportivo, la relajación es recomendada y utilizada como estrategia para ayudar al individuo a lidiar con cualquier estrés físico, psicológico y social, producto del entrenamiento pesado, escuela y situaciones en el hogar (Lima et al., 2019). Siendo recomendada como una de las actividades planeadas para el desarrollo del deportista a largo plazo ( Swimming/Natation Canada, 2008). Antecedentes indican la existencia de varios direccionamientos con resultados positivos para el uso de la relajación en el deporte de competición y sugieren incorporar la práctica regular de la relajación para obtener beneficios físicos y mentales (Mikicin \& Kowalczyk, 2015, Scott et al., 2016).

Sin embargo, el estado actual de la investigación en esta área, desafortunadamente, puede ser compleja y con severas limitaciones debido a la diversidad de técnicas y el hecho de que un mismo método puede mostrar diferentes formas de presentación, versiones o derivaciones. Además, de acuerdo con la citación de Payné: "Cualquier medición de la relajación se verá afectada en cierta medida por el efecto placebo" (2010, p. 337).

Debido a la variedad de técnicas existentes y utilizadas, resulta necesario clasificarlas según el estímulo inicial, es decir, del campo o área en que la persona enfoca su atención para relajarse conforme Bousingen (1997), Payné (2010), Smith (1999) y Undurraga (1994). Inicialmente, el Método Somático - Analítico o Sistema Físico, el punto inicial es fisiológico y el factor biológico se acentúa con el control muscular contracción-relajación. El método ejemplo es la Relajación Progresiva de Jacobson (Payné, 2010, Smith, 1999, Undurraga, 1994). En segundo lugar, el método Cognitivo o Global o Sistema Mental se destaca el papel psicológico y una concepción global de la personalidad humana. El Entrenamiento Autógeno de Schultz es un ejemplo de esta vertiente (Payné, 2010, Smith, 1999, Undurraga, 1994). Para finalizar, el método Sensorial externo corresponde a estímulos externos sonoros, vocales o auditivos y visuales. Como ejemplo, escuchar música, mantras, sonidos de la naturaleza, mirar paisajes, objetos religiosos, entre otros (Smith, 1999).

En ese sentido, precisamente este estudio de Revisión Teórica tuvo como objetivo conocer el uso de métodos de relajación en deportistas y las evidencias de eficacia en el proceso de entrenamiento y/o competiciones. Conjuntamente, se procuró conocer los deportes y participantes, la duración de las intervenciones y resultados principales.

\section{ANTECEDENTES}

Smith (1999) presenta más de 60 aplicaciones y una vasta utilidad del uso de la relajación. Las técnicas de relajación son utilizadas para inducir el sueño, reducir el dolor y tranquilizar las emociones con el objetivo común de producir en el cuerpo conscientemente una respuesta natural de relajación, que se caracteriza por una respiración más lenta, presión arterial baja y una sensación de calma y bienestar (NCCAM, 2011).

En el deporte, la relajación es recomendada y utilizada como estrategia para ayudar al individuo a lidiar con cualquier estrés físico, psicológico y social que resulte del entrenamiento pesado, escuela y 
situaciones en el hogar (Lima et al., 2019, Singer, 1982).

Los atletas citan la relajación, entre otras técnicas (ej. auto-habla, estiramiento), como una estrategia personal y mental para enfrentar la presión, disminuir la tensión y mejorar el rendimiento (González et al., 2016). La utilización es de carácter individual, situacional. Además, el tiempo invertido en la relajación depende de la técnica utilizada (Holland et al., 2010, Kudlackova et al., 2013, Maniar et al., 2001, Stevens \& Lane, 2001).

Walsh (2011) agrega la utilización de la relajación para disminuir el estrés de atletas en rehabilitación. Siendo, además, recomendada como una de las actividades planeadas para el desarrollo del deportista a largo plazo a partir de los 11 años (Calder, 2007, Swimming/Natation Canada, 2008).

En estudios realizados con atletas de diferentes deportes y niveles de competición, la relajación fue considerada por los atletas como relevante para el rendimiento ya que la auto utilizan como una habilidad psicológica para combatir el estrés competitivo, ansiedad, inducir el sueño, recuperación y para regular el humor (Fletcher \& Hanton, 2001, Juliff et al., 2015, Kudlackova et al., 2013, McDonough et al., 2013, Stevens \& Lane, 2001).

En esa perspectiva, es posible sintetizar la idea de que esta investigación explora los detalles (en torno/relativos al uso de la relajación) que rodean el uso de la relajación, observando los efectos que puedan existir en las personas que practican el deporte de competición.

\section{DESARROLLO}

La metodología utilizada tuvo como base el descrito por Liberati et al. (2009). Inicialmente, se utilizó la combinación Booleano/Frase de las siguientes palabras claves en inglés: relaxation therapy $O R$ relaxation technique) AND (athlete OR athletic participation $O R$ athletic performance $O R$ competition OR training) NOT (disability OR disabilities OR disabled). Una vez realizada la lectura de los títulos y resúmenes, se han observado escasos artículos. Por esta razón se han efectuado nuevas combinaciones de palabras como visualización (visualization), meditación (meditation), mindfulness, yoga y sofrología (sophrology). Se han utilizado como interface para la búsqueda el EBSChost con las bases de datos electrónicos: MEDLINE y SPORTDiscus with Full Text. Fueron rastreados los artículos académicos escritos en inglés, español y portugués. Admitidos con limitación de franja etaria entre 13 hasta 44 años, comprehendiendo la literatura desde 2000 hasta marzo de 2019.

Se han considerado como criterios de inclusión que el trabajo debería ser experimental o cuasi experimental, contener control de variables y presentar resultados cualitativos y/o cuantitativos. Además, los participantes deberían ser deportistas de competición de deportes colectivos o individuales. No hubo limitación por tipo o número de técnicas de relajación utilizadas.

Como criterios de exclusión fueron rechazados: estudios que no presentaron instrumentos utilizados, que eran revisión bibliográfica o análisis sistemático de literatura o que sólo fueron publicados en actas de congresos. Además, se excluyeron aquellos estudios en los que la relajación estaba incorporada a un programa conjunto con otras técnicas no consideradas de relajación, por ejemplo, el entrenamiento en habilidades psicológicas que incluye auto-habla (PST Psychology Skill Training).

Finalmente, las informaciones son presentadas de acuerdo con: características de los participantes (número de sujetos, deporte practicado, franja etaria); método o técnica utilizada; duración de la intervención; tipo de estudio; resultados.

\section{PROPUESTA}

Una vez realizado la revisión de los títulos y resúmenes, se ha observado escasos artículos. Cabe destacar, también, la duración de las intervenciones. Se han encontrado predominantemente intervenciones con un tiempo relativamente corto. 
No obstante, también se han hallado estudios más prolongados de seis meses (Haney, 2004), de una temporada completa (Bernier et al., 2009), e incluso hasta alcanzar dos años de duración (Raju et al., 1994).

Se inicia, con el estudio de Raju et al. (1994), en el que observaron los efectos del yoga en atletas con análisis de marcadores físicos y biológicos. El estudio constó de dos fases de la intervención, con uno y dos años de duración respectivamente, y entre sus conclusiones señalaron mejores indicadores en la frecuencia respiratoria, de consumo de oxígeno, y de niveles de lactato del GE en relación con el grupo control.

En el estudio de Patil et al. (2013), participaron 24 ciclistas (11 a 13 años) con el objetivo de observar el efecto del entrenamiento de yoga en el estrés. El grupo fue aleatoriamente dividido: el GE con 12 sujetos recibían entrenamiento de yoga diariamente durante 4 semanas mientras que, el GC con otros 12 sujetos, continuaron con su práctica habitual con la misma duración. Utilizaron instrumentos para evaluar el estrés, la variabilidad de frecuencia cardiaca y la presión arterial. Al final del estudio, concluyeron que la práctica de yoga ayuda a reducir el estrés mediante la optimización de las funciones cardiacas y sugieren incorporar la práctica regular de yoga para obtener beneficios físicos y mentales.

El uso del yoga también estuvo presente en los estudios con intervención en nadadores. El yoga es una práctica milenaria y posee diversas modalidades. No es el objeto del presente estudio profundizar sobre los detalles de cada modalidad de la técnica de yoga, pero sí observar los efectos en el deporte. De esta manera pasa a comentar el estudio de Briegel et al. (2013) con nadadores. En este caso, el objetivo del estudio fue observar los efectos de una intervención con yoga en el mindfulness y flow de jóvenes nadadores. En lo referente a mindfulness o Atención Consciente o Atención Plena es definido por Kabat-Zinn como: "la conciencia que surge al prestar atención, en el momento presente y sin perjuicios al desarrollo de la experiencia momento a momento" (2003, p.145, en Briegel et al., 2013). A su vez, flow se refiere a un estado psicológico óptimo que implica una absorción completa en la tarea o actividad. Fue definido por Csikszentmihalyi como "un sentimiento de completa armonía, absorción total con la actividad realizada de forma tal que la motivación intrínseca y el placer de realizarla es la principal fuerza que dirige la participación" (como se citó en Macías, 2014, p. 11).

Retornando al estudio de Briegel et al. (2013) para lograr los objetivos contaron con la participación de 21 nadadores con edad media de 13 años. Los participantes fueron divididos en dos grupos, Grupo Experimental (GE) y Grupo Control (CC) y evaluados con la escala CAMM (Child and Adolescent Mindfulness Measure, Greco et al. (2011), la escala de estado de fluidez (DFS-2, Jackson et al. (2008) y realizaron una encuesta cualitativa diseñada específicamente para el estudio. Semejante a este estudio, al GE se adhirió una hora por semana de sesión, en este caso, de yoga mientras el GC, continuó con sus actividades normales. Diferente a este estudio, las sesiones fueron en grupo y todos fueron inmediatamente después del entrenamiento. Las sesiones de yoga perduraron por 10 semanas. Los resultados indicaron que la intervención no presentó efecto significativo en el nivel de mindfulness y flow en los participantes de los dos grupos. Por otra parte, en los datos cualitativos estuvieron presentes varias percepciones positivas en temas cognitivos (p. ej. bienestar, concentración, motivación), de rendimiento (p. ej. mejor técnica de nado) y fisiológicos (p. ej. facilidad para dormir) lo que indica la importancia de abordar los efectos de una intervención con aspectos cualitativos y cuantitativos.

En el estudio de Brunelle et al. (2015) el yoga hacia parte de la preparación de los atletas de patinaje. La intervención de 8 semanas contó con la participación de 15 atletas (media de edad de 21 años) y ocupó el 30\% del tiempo del entrenamiento. Como instrumentos de análisis fueron utilizados antes y después de la intervención test de posturas 
con la percepción esquemática del cuerpo y el tiempo para realizar tareas motoras. Además, fueron observadas las lesiones y asistencia al entrenamiento. Los resultados identificaron significante mejora de las posturas corporales y los entrenadores observaron que, a pesar de haber utilizado parte del tiempo de entrenamiento, las sesiones no perjudicaron el rendimiento deportivo. Incluso, no se notó faltas a los entrenamientos por motivos de trauma o malestar de este. Podemos concluir que el tiempo invertido con las sesiones de yoga no fue un estorbo para el entrenamiento, sino que perfeccionó las posturas y redujo las lesiones.

Nuevamente, el impacto del yoga en atletas fue tema de estudio de Polsgrove et al. (2016). Los autores observaron la flexibilidad y el equilibrio para mejorar el rendimiento de jugadores de baloncesto. Al final de 10 semanas de intervención, comparando con el GC, encontraron un incremento de las dos variables en el GE y, además, no encontraron cambios en el GC.

En el estudio de Goodman et al. (2014) la intención fue de explorar la utilidad, viabilidad y efectividad de una intervención que conjugaba mindfulness y yoga, en el bienestar de 13 atletas (edad media de 20 años) la intervención con atletas universitarios consistía de 8 sesiones. El grupo fue comparado con otros 13 sujetos que no fueron intervenidos. Diversos instrumentos han sido utilizados: el Mindful Attention and Awareness Scale (MAAS; Brown y Ryan de 2003) para medir la frecuencia de estados de atención plena durante la vida diaria; el Tolerance of Negative Affect States Scale (TNASS; Bernstein y Brantz de 2012) para observar la tolerancia a los afectos negativos; el Adult Hope Scale (AHS; Snyder et al. de 1991) para medir la esperanza; el Perceived Stress Scale (PSS; Cohen, Kamarck, y Mermelstein de 1983) para evaluar la cantidad de estrés percibido durante el mes; el Valued Living Questionnaire (VLQ; Wilson et al. de 2011) para evaluar el compromiso con los valores; el

1 El entrenamiento de mindfulness busca la capacidad de atender una tarea sin distracciones por medio de la meditación (Mrazek et al., 2014).
Short Grit Scale (Grit-S; Duckworth y Quinn de 2009) para evaluar en el área psicológica, la perseverancia y rasgos de pasión en objetivos a largo plazo; el Depression Anxiety Stress Scale (DASS-21; Henry y Crawford de 2005) para evaluar la angustia psicológica y; un feedback escrito para evaluar el programa. Como resultados destacamos que, en comparación al GC, el GE presentó un mayor nivel de mindfulness, energía dirigida a los objetivos, percibieron menor nivel de estrés y tuvieron una mayor tolerancia a las experiencias negativas al final de la intervención. Lo que indica que la intervención tuvo un efecto positivo sobre los atletas.

Otro de los métodos de relajación que se encuentra presente en estudios es el de la denominada Relajación Progresiva (RP) de Jacobson, creada en 1934, si bien es preciso matizar que también, como ocurre con otros métodos, se han desarrollado distintas corrientes o enfoques de aplicación. La RP, con la intención de regular el estrés, se utiliza para suprimir progresivamente las tensiones musculares a fin de provocar una tranquilidad mental (Mercado, 1984).

Así, la RP fue utilizada, por ejemplo, en el estudio de Jithendra \& Shahin (2014). El estudio contó con la participación de 40 jugadores de cricket con menos de 18 años. La investigación observó y reveló que la intervención proporcionó una mejora positiva en las relaciones entre los jugadores del equipo que participaron del entrenamiento con la relajación.

En el caso del estudio de Bara et al. (2002), la intervención con RP buscó conocer el efecto del método en los niveles del cortisol sanguíneo de nadadores. Se utilizó el análisis de medida fisiológica del cortisol sanguíneo, en diferentes fases del entrenamiento (pretest, mid-test y post-test). Los resultados demostraron que, en comparación con el GC, el GE presentó reducción del nivel de cortisol sanguíneo, concluyéndose que la relajación progresiva es eficaz en la reducción del cortisol 
sanguíneo. La idea central es que el alto nivel de cortisol sanguíneo sugiere depresión, pérdida de control y estrés (Díaz et al., 2013).

Asimismo, en el marco de las intervenciones con RP, Sharifah \& Hashim (2016) compararon dos protocolos del método que utilizan 7 o 16 grupos musculares en la práctica del método y analizaron el ritmo cardíaco, el VO2 máx. y tiempo de reacción en futbolistas. Al final, encontraron que el método que afecta a más grupos musculares, y que por tanto también presenta una mayor duración en cada sesión práctica, es más eficiente para reducir el ritmo cardiaco y mejora en tiempo de reacción rápida.

Igualmente, llama la atención, en el estudio de Haney (2004), que examinó los efectos de una intervención de manejo del estrés utilizando la RP basada en Bernstein \& Borkovec (1973). 47 atletas con edad entre 16 y 51 años participaron en 6 grupos de manejo de estrés (3 cognitivos y 3 relajación) durante 6 meses. Fue utilizada la escala autoeficacia general de Sherer et al. (1982); el inventario de rasgos de ansiedad de Spielberger et al. (1970) y; para medir el afrontamiento, la escala COPE de Carver et al. (1989). Los resultados de la intervención post intervención indicaron una mejora significativa de autoeficacia, reducción de la ansiedad y enfrentamiento adaptativo. Del estudio, Ilama la atención que se ha observado un significativo incremento de la ansiedad en los participantes de relajación durante el periodo de seguimiento después de 2 meses.

En la búsqueda de estudios vinculados con la relajación y el deporte, también se encuentran referencia a la técnica denominada embodied relaxation. En embodied relaxation la técnica consiste, en términos generales, en ejecutar movimientos rítmicos oscilando entre tensión y relajación muscular con atención a la respiración. Ottoboni et al. (2014) utilizaron esta técnica semanalmente (duración de 30min) con 7 atletas (edad entre 13 y 45 años) de Taekwondo durante 7 semanas y los comparó con 9 atletas (GC, edad entre 13 y 32 años) que realizaban entrenamiento estándar adicional. Para evaluar el rendimiento han sido analizados los colores del cinturón del Taekwondo en examen de graduación. Los resultados no indicaron significativas diferencias en el rendimiento, aunque fue mejor el grupo que realizó la intervención.

Ya en el estudio de Bhambri et al. (2005) se han comparado tres grupos de relajación general, visualización y un combinado de las dos técnicas sobre los efectos en dimensiones de la fuerza mental de los jugadores de tenis de mesa. En el caso de la relajación general utilizada en la intervención, el investigador solicitaba al deportista relajar los músculos, uno por uno, desde la cabeza a los pies. Al final de la intervención observaron ser más eficaz la combinación de las dos técnicas (relajación + visualización) con mejoras en la auto confianza, control de energía negativa, atención, motivación positividad, actitud. En el grupo que sólo había realizado visualización las mejoras fueron en motivación, energía positiva y control. El grupo que había realizado solamente la relajación mejoró en el nivel de auto confianza.

Para terminar con los estudios con visualización, Koehn et al. (2014) utilizaron la técnica en intervención con visualización para mejorar el rendimiento y flow. Realizaron la intervención de 6 semanas con 4 jugadores de tenis con edad entre 13 y 15 años. Las sesiones fueron de 3 veces por semana con duración de 10 a 15 minutos y, además, los participantes fueron instruidos a utilizar la visualización 15 minutos antes de la competición. Como instrumentos de evaluación se utilizó el Sport Imagery Ability Measure (SIAM; Watt et al.de 2004) para medir la habilidad de visualización en el deporte; el Flow State Scale-2 (FSS-2; Jackson \& Eklund, 2002) para observar la intensidad de flow; el rendimiento en competición; un diario para registrar las experiencias durante las sesiones $y$; una entrevista para evaluar la experiencia. Felizmente, lograron mejoras significativas en flow y rendimiento en 3 de los 4 participantes. Cabe subrayar que todos los participantes opinaron que la 
intervención generó un incremento en la confianza y fue útil para la rutina y en las competiciones.

Un método que en el ámbito de la preparación deportiva trabaja con la combinación de relajación y visualización es la sofrología, método desarrollado por el médico Alfonso Caycedo en la década de los años 60, durante su formación y estancia en Madrid. La sofrología fue utilizada en el estudio de Slimani et al. (2014). Constó con la participación de 120 luchadores de kickboxing que divididos en tres grupos: 40 sujetos fueron sometidos al entrenamiento sofrológico; 40, al entrenamiento nomológico y; 40, el GC sin intervención. Del entrenamiento nomológico la acción cita inicialmente las ideas negativas y posteriormente durante cinco minutos establece un espíritu constructivo para una mayor motivación. Los resultados indicaron una significante reducción del tiempo de reacción y capacidad de retención en los dos grupos de intervención, siendo mejores los que participaron del entrenamiento sofrológico. Lo que justificaría la utilización de las técnicasy, además, los autores sugieren una combinación de las dos para optimizar los efectos.

En cuanto al estudio de Mikicin \& Kowalczyk (2015), se ha utilizado la compilación de dos técnicas de relajación (audio-visualización y autógeno). Por lo que se refiere al entrenamiento autógeno de Schultz, utilizado en la intervención, la técnica procura obtener la relajación mental mediante la supresión de las tensiones musculares. Los autores investigaron los efectos de la intervención que se realizaba después de cada sesión de entrenamiento durante 7 meses. En cuanto a la audio-visualización la estimulación del sujeto ocurre con luz (posición tumbada, ojos cerrados, luz verde, intensidad de luz ajustada individualmente a la sensibilidad visual de los sujetos) y auditivos (tono agudo con intensidad ajustada individualmente y frecuencia alfa).

Proseguimos con Mikicin \& Kowalczyk (2015), el estudio utilizó como medidas de evaluación el Work curve test (Kraepelin de 1922; Arnold de 1975) para medir la velocidad, efectividad y precisión del trabajo. Finalmente, los resultados indicaron que la intervención mejoró significativamente la capacidad de realizar un esfuerzo mental prolongado. Concluyendo que las técnicas conjugadas pueden mejorar el rendimiento durante el entrenamiento y en las competiciones.

También la hipnosis ha estado presente en diversos estudios. La hipnosis, es un procedimiento en el que se utiliza la sugestión para manipular y modificar el foco de la atención de sentimientos, percepciones, comportamientos y memoria. Fue utilizada en estudios de Barker et al. (2010) y Barker \& Jones (2008) con intención de evaluar los efectos en el rendimiento y en la autoeficacia.

El primer estudio, de Barker et al. (2010), contó con la participación de 59 jugadores de futbol con edad media de 21.34 años. 30 de ellos participaron de 3 sesiones con hipnosis. 29 fueron considerados GC $y$ no fueron intervenidos. Todos fueron evaluados antes, después y con un periodo de seguimiento de 4 semanas con: test deportivo para el rendimiento, el Soccer Wall-Volley Self-Efficacy Questionnaire (SWVSEQ; basado en Bandura, 1997) para la autoeficacia; el Perceived Value of Training Form (PVT Form; basado en Jones et al. de 2002) para evaluar y controlar el efecto placebo; Hypnotic Depth Scale (HDS; Sapp y Evanhow de 1998) para indicar el nivel de hipnosis y; para finalizar, un formulario para comentar la intervención. Del GE, los resultados significativos $(p<.005)$ fueron de mejora en la autoeficacia y rendimiento en post test $y$ seguimiento. Al final, los autores sugirieron que la hipnosis ayuda en la confianza.

El segundo estudio, de Barker \& Jones (2008), fue un estudio de caso con un jugador profesional de futbol. En este tema, el participante de 23 años participó de 8 sesiones de aproximadamente 70 min. Una curiosidad fue que el participante realizó la intervención sin comunicar a su superior la participación en el experimento, debido a la posibilidad de ser expulsado del equipo por estar en terapia psicológica. Para evaluar la intervención, se destaca la utilización del Soccer Self-Efficacy Questionnaire (SSEQ; basado en Treasure et al., 1996) para medir la autoeficacia; el Hypnotic Depth Scale 
(HDS; Sapp \& Evanhow, 1998) para indicar el nivel de hipnosis y; el Soccer Performance Measure (SPM; basado en Annesi, 1997) para medir subjetivamente el rendimiento. Los resultados presentaron al final del periodo de intervención, una mejora de $28 \%$ en la autoeficacia y 34\% del rendimiento. Se apreció también que el jugador atribuyó a la intervención una mejora del esfuerzo, tensión, consistencia y nivel deportivo.

El biofeedback fue utilizado en el estudio de Gracz et al. (2007) para mejorar la actuación deportiva y optimización del rendimiento del jugador de tenis. El biofeedback o biorretroalimentación o retroalimentación biológica es una técnica que utiliza instrumentos (sensores y transductores) para demostrar al individuo sus propios estados biológicos involuntarios. Con la participación de 4 jugadores con edades entre 16 y 21 años, se comparó la ejecución y posición en la clasificación nacional. Los resultados demostraron una mejora considerable en la ejecución y en la posición en el ranking después de 8 a 10 sesiones de biofeedback.

El estudio más reciente de Mistretta et al. (2017) utiliza la técnica mindfulness (atención plena). El entrenamiento de Mindfulness, maneja la meditación para mejorar esta capacidad en atender una tarea sin distracciones (Mrazek et al., 2014). El estudio de Mistretta et al. (2017) contó con la participación de 45 atletas de diversos deportes, incluyendo natación, con una edad media de 19.3 años. Los autores tenían como finalidad comprender las expectativas de la intervención con mindfulness. La intervención tuvo una duración de 6 semanas con un encuentro semanal de $75 \mathrm{~min}$ cada domingo por la mañana. Fueron utilizados varios instrumentos: el Depression, Anxiety, and Stress Scales (DASS-21, Henry \& Crawford, 2005, Lovibond \& Lovibond, 1995) para medir trastornos psicológicos; el Satisfaction With Life Scale (SWLS; Diener et al. de 1985) para medir la satisfacción general de vida; el Five-Facet Mindfulness Questionnaire (FFMQ; Baer et al. de 2006) para el estado de atención; el Acceptance and Action
Questionnaire-II (AAQ-II; Bond et al. de 2011) para medir la inflexibilidad psicológica; Dispositional Flow Scale (DFS-2; Jackson \& Eklund, 2002, 2004) para la experiencia de flow; Sport Anxiety Scale (SAS; Smith et al., 1990) para medir la ansiedad antes y durante la competición; el Sport Rating Form (adaptado de Wolanin, 2004 y Hasker, 2010) que evalúan el rendimiento físico, la regulación de la atención/emoción y cohesión y; para finalizar un cuestionario para evaluar el programa de intervención. En los resultados consta que los participantes tenían una expectativa de beneficiarse de la experiencia tanto dentro como fuera del ámbito deportivo incluyendo un mejor rendimiento. Incluso, post intervención, los participantes mencionaron que experimentaron beneficios psicológicos relacionados al deporte y su vida cotidiana como reducción del estrés y las preocupaciones.

Anteriormente, mindfulness fue utilizado en el estudio de Scott et al. (2016). La investigación buscó los efectos de ocho semanas de entrenamiento con mindfulness en la ansiedad deportiva, pesimismo y flow en 27 ciclistas. Los resultados revelaron efectos positivos en mindfulness, flow, ansiedad deportiva y pesimismo cuando comparados con un grupo control.

De igual modo se ha utilizado mindfulness, en Bernier et al. (2009), con 7 jugadores de golf con edad media de 15,67 años. Se buscó observar la efectividad del entrenamiento de habilidad mental con enfoque en mindfulness y aceptación durante toda una temporada. Se valieron del Ottawa Mental Skills Assessment Tool-3 (OMSAT-3, Durand-Bush et al., 2001) y de entrevistas para evaluar habilidades mentales. Se destaca en el resultado la revelación de que todos los participantes percibieron que mejoraron significativamente en el rendimiento, mientras que, el entrenador consideró que la intervención fue útil y relevante. Asimismo, indicaron mejora en el ranking y en los indicadores de comportamiento necesarios (energía, motivación o focos). 
Para terminar, debemos dejar constancia de los estudios que son realizados en medio líquido con la técnica Floating-REST o Restricted Environmental Stimulation Technique. Ésta es una técnica de relajación donde el individuo flota horizontalmente en un tanque con una solución de agua con 30\% de sulfato de magnesio, sin iluminación y reducción de estímulos auditivos (McAleney \& Barabasz, 1993). La particularidad de la técnica es la sensación de ausencia de peso y el aislamiento sensorial. Para ser más específico, el individuo flota horizontalmente en un tanque, sin iluminación, con reducción de estímulos auditivos y además con aire y agua a la misma temperatura de $34,8^{\circ}$ Celcius.

Suedfeld \& Bruno (1990) observaron la mejora del rendimiento de jugadores de balonmano con la utilización de Flotation-REST y visualización. El estudio contó con la participación de 30 sujetos que fueron divididos en tres grupos con 10 integrantes. Un grupo realizó entrenamientos con visualización, otro Flotation-REST y el tercer grupo no sufrió intervención y fue considerado control. Se utilizaron cuestionarios para medir experiencias atléticas, experiencias de imágenes y predicciones de éxito en tiro libre. Los autores no tenían la intención de comparar las dos técnicas, pero los resultados apuntaron que los participantes del grupo de Flotation-REST fueron significativamente mejores en test de rendimiento que el grupo de visualización. Asimismo, observamos que un día de intervención para la visualización pueda ser corta cuando en los estudios de Bhambri et al. (2005) y Koehn et al. (2014) fueron de 2 y 6 semanas.

Finalizamos con Klockare et al. (2015) que también utilizaron Flotation-REST para observar los efectos en deportistas de atletismo. Participaron de la experiencia 6 atletas con edad entre 17 y 23 años con frecuencia de 2 a 6 sesiones. Se realizaron entrevistas inmediatamente después de las sesiones para obtener datos de la experiencia. El análisis de los relatos de las entrevistas proporcionó información interesante como: para todos los participantes ( $n=6)$ sirvió como aprendizaje sobre la relajación; para 3, ocurrió un aumento de la percepción de la importancia de la relajación; para 5, la experiencia fue un alivio en la vida diaria, experiencia placentera y relajante con mejora del bienestar. Al final, los autores concluyeron que la técnica posee un potencial para la promoción de la salud y manejo del estrés.

\section{CONCLUSIONES}

Con la revisión efectuada, se puede concluir que las respuestas a las técnicas de relajación difieren en proporción y frecuencia de acuerdo con cada individuo y, asimismo, existe la disposición para llevar a una persona a experimentar un estado de relajación.

Además, se debe observar la sistemática de las intervenciones y observar individualidades y necesidades de cada individuo para optimizar el proceso, cuando algunas técnicas necesitan más practica o tiempo para ser eficaz en los efectos.

Otro de los aspectos destacables hace referencia al tipo de investigación, en función de que los autores utilizaran la opción de evaluar la información con datos cualitativos o cuantitativos. En ese sentido, encontramos una prevalencia de estudios de naturaleza cualitativa ( $n=13,59 \%$ ), con una menor presencia de estudios con información de naturaleza cuantitativa ( $n=4,18,1 \%$ ). Asimismo, cabe destacar los estudios mixtos, en los que fueron registrados y analizados datos cualitativos y cuantitativos ( $n=5,22,7 \%)$.

En cuanto a los participantes, cabe reseñar que, de las investigaciones encontradas, solamente en dos estudios los protagonistas fueron exclusivamente nadadores (Bara et al., 2002, Briegel et al., 2013), y sólo Hatzigeorgiadis et al. (2014) utilizaron los resultados de competiciones reales como instrumentos de recaudación de datos.

De igual manera llama la atención el número de participantes en los estudios que estuvo dentro de un rango de 6 a 70 participantes. Uno de los dos motivos que puede ampliar el número de participantes es la posibilidad de hacer la intervención en grupo como el yoga. Diferente de, 
por ejemplo, Flotation-REST que es individual y necesita un aparato específico.

Por lo que se refiere a los métodos, se ha encontrado la utilización de 13 técnicas dentro de la variedad posible. La más frecuente fue el yoga ( $n=5$, 22,7\%; sin especificar el método) seguido de la relajación progresiva ( $n=4,18,1 \%)$. Entre todos, sólo Flotation-REST es realizado en medio líquido.

Se concluye que, en la utilización de las técnicas de relajación, no existe estandarización de los protocolos y hay que tener en cuenta la capacidad de adaptación de las técnicas con la posibilidad de aplicación, cuando se realiza un estudio con deportes y equipos distintos. Por tanto, se puede decir que, no hay una técnica mejor o peor que otra, aunque, se recomienda su aplicación.

\section{LIMITACIONES DEL ESTUDIO}

Se ha encontrado escasez de estudios, una razón que se puede admitir es la dificultad de un investigador para intervenir en un riguroso plan de entrenamiento. Están en juego responsabilidades de técnicos y dirigentes, compromisos de padres y sueños de jóvenes en ser medallistas, aspectos que dificultan, cuando no anulan directamente, las posibilidades de intervención.

Además, se ha observado que, en muchos casos, no se ha detallado la frecuencia, duración y período de las intervenciones. Lo que dificulta observar el impacto de las intervenciones en el desarrollo cotidiano de los atletas. Asimismo, en los que se señala la metodología, se han encontrado hasta 45 minutos todos los días después de los entrenamientos (Mikicin \& Kowalczyk, 2015).

\section{APLICACIONES PRÁCTICAS}

Para el deporte, la utilización de técnicas de relajación posee en su concepción beneficios que son útiles no sólo al bienestar del atleta, sino que también ayuda en la recuperación a nivel físico y psicológico.

Además, la relajación se presenta como un ítem importante para el desarrollo a largo plazo del individuo en el deporte con resultados positivos del uso en el deporte de competición.

Sin embargo, es un área estudios que aún debería ser más estudiado, cuando se observa que, pesar la orientación para el uso, el estudio de campo que examina el impacto de las técnicas de relajación en el desempeño de deportistas es limitado.

\section{REFERENCIAS BIBLIOGRÁFICAS}

Bara-Filho, M. G., Ribeiro, L. C. S., Miranda, R., \& Teixeira, T. (2002). A redução dos níveis de cortisol sanguíneo através da técnica de relaxamento progressivo em nadadores. Revista Brasileira Medicina Esporte, 8(4), 139-143. https://doi.org/10.1590/S151786922002000400002

Barker, J. B., \& Jones, M. V. (2008). The Effects of Hypnosis on Self-Efficacy, Affect, and Soccer Performance: A Case Study. Journal of Clinical Sport Psychology, 2(2), 127-147. https://doi.org/10.1123/jcsp.2.2.127

Barker, J. B., Jones, M. V, \& Greenlees, I. (2010). Assessing the Immediate and Maintained Effects of Hypnosis on Self-Efficacy and Soccer Wall-Volley Performance. Journal of Sport \& Exercise Psychology, 32(2), 243-252. https://doi.org/10.1123/jsep.32.2.243

Bernier, M., Thienot, E., Codron, R., \& Fournier, J. F. (2009). Mindfulness and Acceptance Approaches in Sport Performance. Journal of Clinical Sport Psychology, 3(4), 320-333. https://doi.org/10.1123/jcsp.3.4.320

Bhambri, E., Dhillon, P. K., \& Sahni, S. P. (2005). Effect of psychological interventions in enhancing mental toughness dimensions of sports persons. Journal of the Indian Academy of Applied Psychology, 37(7-2), 65-70. https://docplayer.net/7476000-Effect-ofpsychological-interventions-in-enhancingmental-toughness-dimensions-of-sportspersons.html 
Bousingen, R. D. de. (1997). La Relajación. (3a ed.). Paidotribo.

Briegel-Jones, R. M. H., Knowles, Z., Eubank, M. R., Giannoulatos, K., \& Elliot, D. (2013). A Preliminary Investigation into the Effect of Yoga Practice on Mindfulness and Flow in Elite Youth Swimmers. The sport Psychologist, $\quad$ 27(4), 349-359. https://doi.org/10.1123/tsp.27.4.349

Brunelle, J.-F., Blais-Coutu, S., Gouadec, K., Bédard, É., \& Fait, P. (2015). Influences of a yoga intervention on the postural skills of the Italian short track speed skating team. Open Access Journal of Sports Medicine, 6, 23-35.

https://doi.org/10.2147/OAJSM.S68337

Calder, A. (2007). Recovery and Regeneration for Long-Term Athlete Development. Canadian Sport for Life (p. 11). https://es.scribd.com/document/101646054 /Recovery-and-Regeneration-for-Long-

Term-Athlete-Development

González, G., Valdivia-Moral, P., Zagalaz, J. C., \& Ortega, F. Z. (2016). Visualisation and goalsetting in footballers. Journal of Human Sport and Exercise, 17(2), 279-292. https://doi.org/10.14198/jhse.2016.112.01

Díaz, M. M., Bocanegra, O. L., Teixeira, R. R., Tavares, M., Soares, S. S., \& Espindola, F. S. (2013). The Relationship Between The Cortisol Awekening Response, Mood States, and Performance. Journal of Strength and Conditioning Research, 27(5), 1340-1348. https://doi.org/10.1519/JSC.0b013e318267a61 2

Fletcher, D., \& Hanton, S. (2001). The relationship between psychological skills usage and competitive anxiety responses. Psychology of Sport and Exercise, 2(2), 89-101. https://doi.org/10.1016/s1469-

0292(00)00014-5
Goodman, F. R., Kashdan, T. B., Mallard, T. T., \& Schumann, M. (2014). A brief mindfulness and yoga intervention with an entire NCAA Division | athletic team: An initial investigation. Psychology of Consciousness: Theory, Research, and Practice, 7(4), 339356. https://doi.org/10.1037/cns0000022

Gracz, J., Walczak, M., \& Wilińska, K. (2007). Seeking New Methods of Mental Training in Sport. Studies in Physical Culture \& Tourism, 74(1), 97-102.

https://www.cabdirect.org/cabdirect/abstra ct/20073289096

Greco, L. A., Baer, R. A., \& Smith, G. T. (2011). Assessing mindfulness in children and adolescents: Development and validation of the Child and Adolescent Mindfulness Measure (CAMM). Psychological Assessment, 23(3), 606-614. https://doi.org/10.1037/a0022819

Haney, C. J. (2004). Stress-Management interventions for female athlete: Relaxation and cognitive reestructure. International Journal of Sports Physiology, 35(2), 109-118. https://www.tib.eu/en/search/id/BLSE\%3AR N153367448/Stress-managementinterventions-for-female-athletes/

Hatzigeorgiadis, A., Galanis, E., Zourbanos, N., \& Theodorakis, Y. (2014). Self-talk and Competitive Sport Performance. Journal of Applied Sport Psychology, 26(1), 82-95. https://doi.org/10.1080/10413200.2013.79009 5

Holland, M. J. G., Woodcock, C., Cumming, J., \& Duda, J. L. (2010). Mental Qualities and Employed Mental Techniques of Young Elite Team Sport Athletes. Journal of Clinical Sport Psychology, $\quad$ 4(1), 19-38. https://doi.org/10.1123/jcsp.4.7.19

Jackson, S. A., Martin, A. J., \& Eklund, R. C. (2008). Long and short measures of flow: The construct validity of the FSS-2, DFS-2, and new brief counterparts. Journal of Sport \& Exercise Psychology, 30(5), 561-587. https://doi.org/10.1123/jsep.30.5.561 
Jithendra, V. S., \& Shahin, A. (2014). Effect of Jacobson's Deep Muscle Relaxation On Sports Team Relationship Of Under-18 Cricketers. International Journal of Sports Sciences and Fitness, 4(1), 82-88. http://ijssf.org/PDF/v04issue0labs07.pdf

Juliff, L. E., Halson, S. L., \& Peiffer, J. J. (2015). Understanding sleep disturbance in athletes prior to important competitions. Journal of Science and Medicine in Sport, 18(1), 13-18. https://doi.org/10.1016/j.jsams.2014.02.007

Klockare, E., Gustafsson, H., Davis, P., \& Lundqvist, C. (2015). Track and field athletes' experiences and perceived effects of flotation-REST: An interpretative phenomenological analysis. International Journal of Sport Psychology, 46(5),

https://doi.org/10.7352/IJSP.2015.46.409

Koehn, S., Morris, T., \& Watt, A. P. (2014). Imagery Intervention to Increase Flow State and Performance in Competition. The Sport Psychologist, 28(1), 48-59. https://doi.org/10.1123/tsp.2012-0106

Kudlackova, K., Eccles, D. W., \& Dieffenbach, K. (2013). Use of relaxation skills in differentially skilled athletes. Psychology of Sport and Exercise, $\quad$ 14(4), 468-475. https://doi.org/10.1016/j.psychsport.2013.01.0 07

Liberati, A., Altman, D. G., Tetzlaff, J., Mulrow, C., Gøtzsche, P. C., Ioannidis, J. P. A., Clarke, M., Devereaux, P. J., Kleijnen, J., \& Moher, D. (2009). The PRISMA statement for reporting systematic reviews and metaanalyses of studies that evaluate health care interventions: Explanation and elaboration. PLoS Medicine, 6(7), 28. https://doi.org/10.1371/journal.pmed.100010 O
Lima-Costa, A. C., Sanz-Arribas, I., \& HernándezÁlvarez, J. L. (2019). Efectos De La Intervención Con El Método Watsu En Jóvenes Nadadores De Competición Brasileños: Asistencia, Necesidades $Y$ Efectos Experimentados. Revista de Investigación En Actividades Acuáticas, 3(6), 53-59. https://doi.org/10.21134/riaa.v3i6.377

Macías, M. Á. F. (2014). Flow en corredores de maratón, futbolistas, nadadores y jugadores de rugby. [Tesis doctoral]. Universidad Pablo de Olavide. https://dialnet.unirioja.es/servlet/tesis?codi go $=102528$

Maniar, S. D., Curry, L. A., Sommers-Flanagan, J., Walsh, J. A., Curry, L. A., Sommers-Flanagan, J., \& Walsh, J. A. (2001). Student-Athlete Preferences in Seeking Help When Confronted with Sport Performance Problems. The Sport Psychologist, 15(2), 205-223. https://doi.org/10.1123/tsp.15.2.205

McAleney, P., \& Barabasz, A. (1993). Effects of Flotation REST and Visual Imagery on Athletic Performance: Tennis. In A. F. Barabasz \& M. Barabasz (Eds.), Restricted Environmental Stimulation (pp. 79-86). Springer. https://doi.org/10.1007/978-14684-8583-7_10

McDonough, M. H., Hadd, V., Crocker, P. R. E., Holt, N. L., Tamminen, K. A., \& Schonert-Reichi, K. (2013). Stress and Coping Among Adolescents Across a Competitive Swim Season. The Sport Psychologist, 27(2), 143155. https://doi.org/10.1123/tsp.27.2.143

Mercado, C. (1984). Preparación psico-física en deportistas. Técnicas de relajación. Apunts Educació Física y Deportes, 21(82), 103-108. https://www.apunts.org/en-pdfX0213371784048602 
Mikicin, M., \& Kowalczyk, M. (2015). Audio-Visual and Autogenic Relaxation Alter Amplitude of Alpha EEG Band, Causing Improvements in Mental Work Performance in Athletes. Applied Psychophysiology Biofeedback, 40(3),

https://doi.org/10.1007/s10484-015-9290-0

Mistretta, E. G., Glass, C. R., Spears, C. A., Perskaudas, R., Kaufman, K. A., \& Hoyer, D. (2017). Collegiate Athletes' Expectations and Experiences With Mindful Sport Performance Enhancement. Journal of Clinical Sport Psychology, 17(3), 201-221. https://doi.org/10.1123/jcsp.2016-0043

Mrazek, M. D., Broadway, J. M., Phillips, D. T., Franklin, M. S., Mooneyham, B. W., \& Schooler, J. W. (2014). Mindfulness: An Antidote for Wandering Minds. In A. le, C. T. Ngnoumen, \& E. J. Langer (Eds.), The Wiley Blackwell Handbook of Mindfulness (pp. 153-167). John Wiley \& Sons. https://doi.org/10.1002/9781118294895.ch8

NCCAM, C. (2011). Relaxation Techniques for Health: An Introduction. National Institutes of Health.

https://www.nccih.nih.gov/health/relaxatio n-techniques-for-health

Ottoboni, G., Giusti, R., Gatta, A., Symes, E., \& Tessari, A. (2014). Just do it: Embodied experiences improve Taekwondo athletes sport performance. Sensoria: A Journal of Mind, Brain \& Culture, 10(1), 28-33. https://doi.org/10.7790/sa.v10i1.386

Patil, S. G., Mullur, L. M., Khodnapur, J. P., Dhanakshirur, G. B., \& Aithala, M. R. (2013). Effect of yoga on short-term heart rate variability measure as a stress index in subjunior cyclists: a pilot study. Indian Journal of Physiology and Pharmacology, $57(2)$

153-158.

https://www.ijpp.com/IJPP\%20archives/201

3_57_2_Apr\%20-\%20Jun/153-158.pdf
Payné, R. A. (2010). Técnicas de Relajación. Guía práctica para el profesional de la salud. (3a Ed.). Paidotribo.

Payné, R. A. (2010). Técnicas de Relajación. Guía práctica para el profesional de la salud. Paidotribo.

Polsgrove, M. J., Eggleston, B. M., \& Lockyer, R. J. (2016). Impact of 10-weeks of yoga practice on flexibility and balance of college athletes. International Journal of Yoga, 9(1), 27-34. https://doi.org/10.4103/09736131.171710

Raju, P. S., Madhavi, S., Prasad, K. V. V, Reddy, M. V, Reddy, M. E., Sahay, B. K., \& Murthy, K. J. R. (1994). Comparison of effects of yoga \& physical exercise in athletes. The Indian Journal of Medical Research, 100(8), 81-87. https://pubmed.ncbi.nIm.nih.gov/7927562/

Scott-Hamilton, J., Schutte, N. S., \& Brown, R. F. (2016). Effects of a Mindfulness Intervention on Sports-Anxiety, Pessimism, and Flow in Competitive Cyclists. Applied Psychology: Health and Well-Being, 8(1), 85-103. https://doi.org/10.1171/aphw.12063

Sharifah Maimunah, S. M. P., \& Hashim, H. A. (2016). Differential Effects of 7 and 16 Groups of Muscle Relaxation Training Following Repeated Submaximal Intensity Exercise in Young Football Players. Perceptual and Motor Skills, 122(7), 227-237. https://doi.org/10.1177/0031512515625383

Singer, R. N. (1982). Thought Processes and Emotions in Sport. The Physician and Sportsmedicine, $\quad$ 10(7), 75-88. https://doi.org/10.1080/00913847.1982.11947 273

Slimani, M., Hentati, A., Bouazizi, M., Boudhiba, D., Amar, I. Ben, \& Chéour, F. (2014). Effects of Monological and Sophrological Trainings on the Development of Attention and Retention in Tunisian Male Kick Boxers. IOSR Journal of Humanities and Social Science, 19(5), 23-25. https://doi.org/10.9790/0837-19512325 
Smith, J. C. (1999). ABC Relaxation Theory. An Evidence Based Approach. Springer Publishing https://psycnet.apa.org/record/1999-04090000

Stevens, M. J., \& Lane, A. M. (2001). Mood-Regulating Strategies Used By Athletes. Athletic Insight, $\quad 3(3), \quad 1-12$. http://www.athleticinsight.com/Nol3Iss3/M oodPDF.pdf

Suedfeld, P., \& Bruno, T. (1990). Flotation REST and Imagery in the Improvement of Athletic Performance. Journal of Sport and Exercise Psychology, 12(1), 82-85. https://doi.org/10.1123/jsep.12.1.82
Swimming/Natation Canada. (2008). Long term athlete development strategy. Swimming Canada. https://swimming.ca/content/uploads/2015 /06/ltad_en.pdf

Undurraga, C. (1994). Las relajaciones. Psykhe, 3(1), $27-34$. http://www.revistaaisthesis.uc.cl/index.php/ psykhe/article/view/20119

Walsh, A. E. (2011). The Relaxation Response: A Strategy to Address Stress. International Journal of Athletic Therapy \& Training, 16(2), 20-23. https://doi.org/10.1123/ijatt.16.2.20

\section{Dirección para correspondencia}

Ana Carolina Lima Costa

Doctora en Ciencias de la Actividad Física y del Deporte.

Secretaria Ejecutiva de Deporte del Estado de Pernambuco.

Madrid, España.

Dirección Postal: Rua Izabel Magalhães, 51.

Boa Viagem. Recife-PE. 51030-330. Brasil.

ORCID ID: https://orcid.org/0000-0003-2959-0625

Contacto:

carolcostabr@gmail.com

Recibido: 03-01-2021

Aceptado: 09-06-2021 